\title{
Ancestry, Plasmodium cynomolgi prevalence and rhesus macaque admixture in cynomolgus macaques (Macaca fascicularis) bred for export in Chinese breeding farms
}

\author{
Xinjun Zhang ${ }^{1 *}$ (D) | Yuhuan Meng ${ }^{2 *}$ | Paul Houghton ${ }^{3}$ | Mingyu Liu ${ }^{2}$ | \\ Sreetharan Kanthaswamy ${ }^{4,5}$ | Robert Oldt ${ }^{4}$ | Jillian Ng$^{1}$ | Jessica Satkoski Trask ${ }^{6}$ | \\ Ren Huang $^{7}$ | Balbir Singh ${ }^{8}$ | Hongli Du ${ }^{2}$ | David Glenn Smith ${ }^{1,5}$
}

${ }^{1}$ Department of Anthropology, University of California, Davis, CA, USA

${ }^{2}$ School of Bioscience and Bioengineering, South China University of Technology, Guangzhou, China

${ }^{3}$ Primate Products, Inc, Immokalee, FL, USA

${ }^{4}$ School of Mathematical and Natural Sciences, Arizona State University (ASU West Campus), Glendale, AZ, USA

${ }^{5}$ California National Primate Research Center, University of California, Davis, CA, USA

${ }^{6}$ Department of Research Compliance \& Integrity, Office of Research, University of California, Davis, CA, USA

${ }^{7}$ Guangdong Key Laboratory of Laboratory Animals, Guangzhou, China

${ }^{8}$ Malaria Research Centre, Universiti Malaysia Sarawak, Kota Samarahan, Sarawak, Malaysia

\section{Correspondence}

Hongli Du, School of Bioscience and Bioengineering, South China University of Technology, Guangzhou, China.

Email: hldu@scut.edu.cn

and

David Glenn Smith, Department of Anthropology, University of California, Davis CA, USA.

Email: dgsmith@ucdavis.edu

Funding information

California National Primate Research Center, Grant/Award Number: RR000169-48; National Center for Research Resources NCRR, Grant/Award Number: RR005090 and RR025781; National Institutes of Health; Universiti Malaysia Sarawak, Grant/Award Number: 01[TD03]/1003/2013[01]

\begin{abstract}
Background: Most cynomolgus macaques (Macaca fascicularis) used in the United States as animal models are imported from Chinese breeding farms without documented ancestry. Cynomolgus macaques with varying rhesus macaque ancestry proportions may exhibit differences, such as susceptibility to malaria, that affect their suitability as a research model.

Methods: DNA of 400 cynomolgus macaques from 10 Chinese breeding farms was genotyped to characterize their regional origin and rhesus ancestry proportion. A nested PCR assay was used to detect Plasmodium cynomolgi infection in sampled individuals.

Results: All populations exhibited high levels of genetic heterogeneity and low levels of inbreeding and genetic subdivision. Almost all individuals exhibited an Indochinese origin and a rhesus ancestry proportion of $5 \%-48 \%$. The incidence of P. cynomolgi infection in cynomolgus macaques is strongly associated with proportion of rhesus ancestry.

Conclusions: The varying amount of rhesus ancestry in cynomolgus macaques underscores the importance of monitoring their genetic similarity in malaria research.
\end{abstract}

KEYWORDS

admixture, ancestry, Macaca fascicularis, Macaca mulatta, Plasmodium cynomolgi 